\title{
Kraichnan's random sweeping hypothesis in homogeneous turbulent convection
}

\author{
Xiaozhou He and Penger Tong \\ Department of Physics, Hong Kong University of Science and Technology, Clear Water Bay, Kowloon, Hong Kong
}

(Received 4 December 2010; published 24 March 2011)

\begin{abstract}
We report an experimental study of the temperature space-time cross-correlation function, $C_{T}(r, \tau)$, in the central region of turbulent Rayleigh-Bénard convection. The measured $C_{T}(r, \tau)$ is found to have the scaling form $C_{T}\left(r_{E}, 0\right)$, where $r_{E}=\left[r^{2}+(V \tau)^{2}\right]^{1 / 2}$ with $V$ being the rms velocity at the center of the convection cell. The experiment confirms the theory by He et al. [Phys. Rev. E 73, 055303(R) (2006)] and demonstrates its applications to homogenous turbulent flows, where the mean flow velocity is zero and Kraichnan's random sweeping hypothesis holds approximately.
\end{abstract}

DOI: 10.1103/PhysRevE.83.037302

PACS number(s): 47.27.eb, 44.25.+f, 05.40.-a

In a recent Rapid Communication [1], we reported an experimental study of the temperature space-time cross-correlation function,

$$
C_{T}(r, \tau)=\langle\delta T(x+r, t+\tau) \delta T(x, t)\rangle_{t} /\left[\left(\sigma_{T}\right)_{1}\left(\sigma_{T}\right)_{2}\right],
$$

in turbulent Rayleigh-Bénard convection, where a fluid layer of thickness $H$ is heated from below and cooled from the top. In the above, $\delta T$ is the local temperature deviation from the mean and $\left(\sigma_{T}\right)_{i}$ is its standard deviation at position $i$. When the Rayleigh number [2] $\mathrm{Ra} \gtrsim 10^{8}$, the convective flow becomes turbulent. The flow in the closed convection cell is known to be inhomogeneous with a large-scale circulation (LSC) across the cell height [3]. In the rotation plane of LSC, the flow has a fly-wheel-like structure with a zero mean at the center and a linearly increasing velocity along the radial direction in the bulk region. After reaching its maximum value near the sidewall, the mean vertical velocity starts to drop quickly and becomes zero at the cell wall.

As a result, the flow field near the sidewall is similar to that of a channel flow with a mean vertical velocity $U_{0}$ and a rms velocity $\sigma_{v} \simeq 0.6 U_{0}$ [3]. In this case, temperature is a passive scalar and follows the local flow [4,5]. Therefore the energy cascade picture can also be used to describe the spectrum of temperature variance [6], and $C_{T}(r, \tau)$ is expected to have the same functional form as the velocity counterpart $C_{v}(r, \tau)$. It was found [1] that the obtained $C_{T}(r, \tau)$ has the scaling form

$$
C_{T}(r, \tau)=C_{T}\left(r_{E}, 0\right),
$$

with $r_{E}$ being of the elliptical form,

$$
r_{E}^{2}=(r-U \tau)^{2}+(V \tau)^{2},
$$

where $U$ is a characteristic convection velocity proportional to the local mean velocity $U_{0}$ and $V$ is associated with a random sweeping velocity proportional to the local rms velocity $\sigma_{v}$. Equation (3) incorporates both Taylor's frozen flow hypothesis [7,8] when $V$ is small and Kraichnan's random sweeping hypothesis [9] for a homogenous and isotropic turbulent flow with a zero mean velocity. The experiment thus verified the theory by He and Zhang [10], in which Eq. (3) was derived for small values of $r$ and $\tau$ and both $U$ and $V$ were calculated from the second derivatives of $C_{v}(r, \tau)$.

The scaling theory by He and Zhang [10] has important practical implications for a large class of turbulent flows and thus it is essential to test the theory in different flow systems. The above experiment tested the theory in the sidewall region of a convective flow, where there still exists a dominant mean flow but the rms velocity is so large that Taylor's hypothesis [7] does not hold. In the experiment, $r$ was varied only in the longitudinal direction along the mean flow. In this Brief Report, we present new measurements at the center of the convection cell, where the mean flow is zero and velocity fluctuations are approximately homogeneous [3]. Here we vary $r$ in the lateral direction across a large-scale shear imposed by LSC. The experiment further confirms the theory by $\mathrm{He}$ and Zhang and demonstrates its applications to homogenous turbulent flows, where Kraichnan's hypothesis [9] holds approximately.

The experiment is conducted in an upright cylindrical cell of inner diameter $D=19.0 \mathrm{~cm}$ and height $H=22.0 \mathrm{~cm}$ filled with water. Details about the apparatus and the experimental method have been described elsewhere [11], and here we mention only some key points. The sidewall of the cell is made of a transparent Plexiglas ring, which is sandwiched between the top and bottom brass plates. Two silicon rubber film heaters are used to provide uniform heating to the bottom plate. The top plate temperature is maintained constant by a temperature bath. The entire cell is placed inside a thermostat box, whose temperature matches the mean temperature of the bulk fluid, which is maintained at $40 \pm 0.3{ }^{\circ} \mathrm{C}$. The Prandtl number is then fixed at $\operatorname{Pr} \simeq 4.3$. The value of $\mathrm{Ra}$ is varied in the range of $9 \times 10^{8} \lesssim \mathrm{Ra} \lesssim 2 \times 10^{10}$, and here we focus on the measurements at $\mathrm{Ra}=2 \times 10^{10}$.

Two small movable thermistors of $0.2 \mathrm{~mm}$ in diameter and $15 \mathrm{~ms}$ in time constant are used to measure temperature fluctuations at two positions simultaneously. One of the thermistors is fixed at the cell center and the other is placed aside at the same height with a horizontal separation $r$, which is varied from 0.1 to $90 \mathrm{~mm}$. For each value of $r$, we collect 10-h-long time series data ( $>10^{6}$ data points) to ensure that the statistical averaging is adequate. All the temperature measurements are made in the plane of LSC with a fixed sampling rate of $40 \mathrm{~Hz}$. Because of the central rotation symmetry of LSC, care is taken to align the position of the thermistors at an accuracy better than $30 \mu \mathrm{m}$.

Figure 1(a) shows a three-dimensional (3D) plot of the measured $C_{T}(r, \tau)$ as a function of $r$ and $\tau$. The measured 

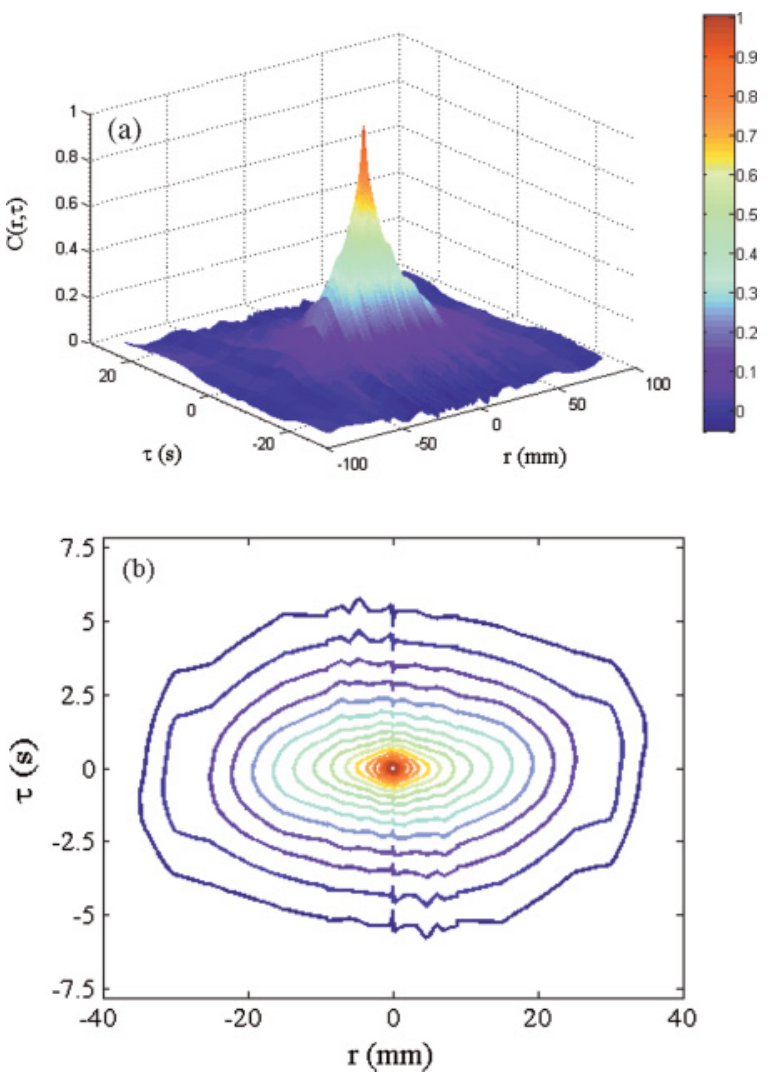

FIG. 1. (Color online) (a) 3D plot of the measured $C_{T}(r, \tau)$ as a function of $r$ and $\tau$. The correlation amplitude is color coded. (b) $2 \mathrm{D}$ plot of isocorrelation contours of $C_{T}(r, \tau)$ with the correlation amplitude varied from 0.95 to 0.2 at decrements of 0.05 (inner to outer contours). All the temperature measurements are made in the central core region at $\mathrm{Ra}=2 \times 10^{10}$.

$C_{T}(r, \tau)$ is a single peaked function with a maximal value of $C_{T}(0,0)=1$ at the origin and decays to zero at large values of $r$ and $\tau$. Figure 1(b) shows a 2D plot of isocorrelation contours of $C_{T}(r, \tau)$. It is found that for small values of $r(\lesssim 10 \mathrm{~mm}$, inner region), the obtained isocorrelation contours appear as a set of ellipses of the standard form having the same orientation and aspect ratio. The long and short major axes of the ellipses coincide with the $r$ and $\tau$ axes, respectively. For larger values of $r$ ( $\gtrsim 10 \mathrm{~mm}$, outer region), the isocorrelation contours start to deviate from the standard form of ellipse. The deviations are found to increase with increasing $r$. Clearly, the functional form of $C_{T}(r, \tau)$ in the outer region is different from that in the inner region.

We first discuss the scaling form of $C_{T}(r, \tau)$ in the inner region. According to the elliptic model by He and Zhang [10], the set of elliptic contours shown in Fig. 1(b) results from the fact that $C_{T}(r, \tau)$ has the scaling form shown in Eqs. (2) and (3). The values of $U$ and $V$ in Eq. (3) can be obtained directly from the measured $C_{T}(r, \tau)$. Figure 2(a) shows the measured $C_{T}(r, \tau)$ as a function of $\tau$ for different values of $r$. It is seen that $C_{T}(r, \tau)$ has a symmetric shape with the peak position remaining at $\tau_{p}=0$ for all values of $r$ in the inner region. This behavior is in great contrast with that near the sidewall, where $\tau_{p}$ was found to increase with $r$ [1]. This is because it takes a longer time for temperature fluctuations to
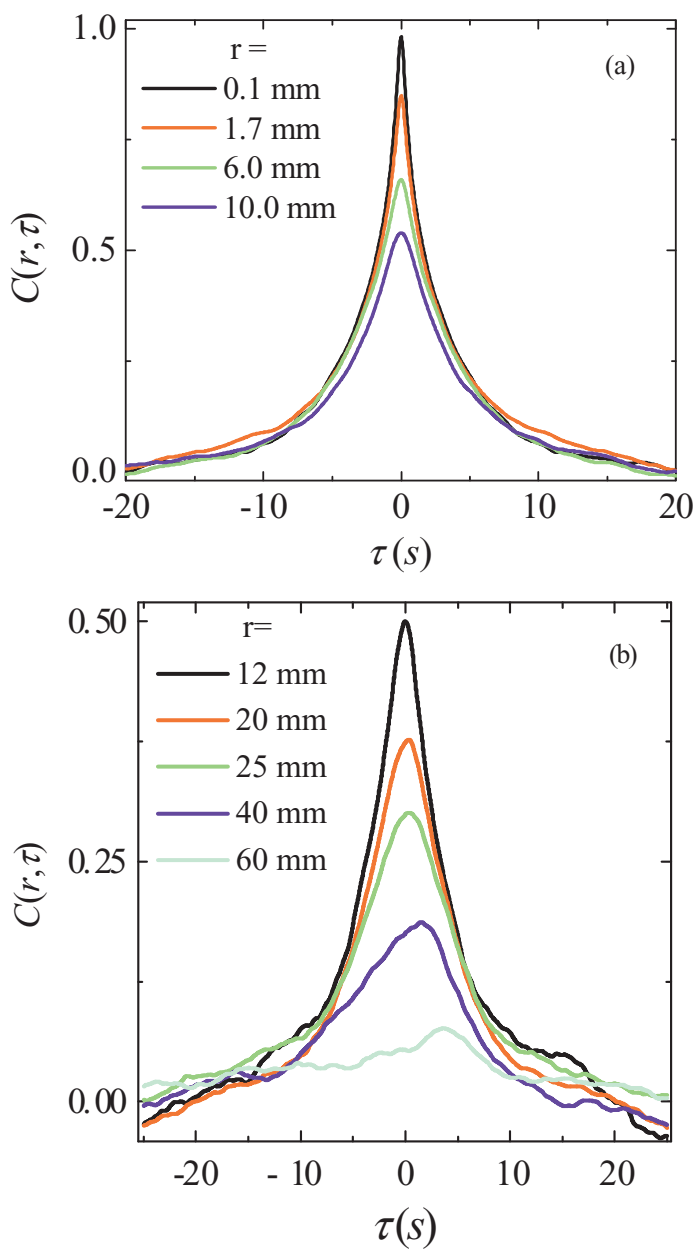

FIG. 2. (Color online) Measured $C_{T}(r, \tau)$ as a function of $\tau$ for different values of $r$. Values of $r$ (from top to bottom): (a) $0.1 \mathrm{~mm}$ (black), $1.7 \mathrm{~mm}$ (red), $6.0 \mathrm{~mm}$ (green), $10.0 \mathrm{~mm}$ (blue); (b) $12.0 \mathrm{~mm}$ (black), $20.0 \mathrm{~mm}$ (red), $25.0 \mathrm{~mm}$ (green), $40.0 \mathrm{~mm}$ (blue), and $60.0 \mathrm{~mm}$ (cyan).

move across a larger separation by the mean flow $U$. It has been shown that $\tau_{p}$ is related to $r$ by the equation [1]

$$
\tau_{p}=\frac{U}{U^{2}+V^{2}} r
$$

From Eq. (4) and Fig. 2(a), we conclude that $U=0$ in the inner region. This conclusion is also supported by the fact the long major axis of the elliptic contours coincide with the $r$ axis, as shown in Fig. 1(b). He and Zhang [10] have shown that the angle of the long major axis with respect to the $r$ axis is zero when $U=0$.

Substituting $U=0$ into Eq. (3), one finds that Eq. (3) can be rearranged into a standard form of the ellipse:

$$
\frac{r^{2}}{a}+\frac{\tau^{2}}{b}=1,
$$

where $a=r_{E}$ and $b=a / V$. We have verified that the values of $a$ and $b$ obtained from the isocorrelation contours shown in Fig. 1(b) indeed obey a linear relation (not shown), and from the slope we find $V=8.5 \mathrm{~mm} / \mathrm{s}$. Previous velocity measurements in a similar convection cell [3] have shown that at the cell center the mean horizontal velocity $U_{0}=0$ and the 
horizontal $\mathrm{rms}$ velocity $\sigma_{v}=8 \mathrm{~mm} / \mathrm{s}$. These values are very close to those obtained for $U$ and $V$. Figures 1 and 2(a) thus demonstrate that Kraichnan's random sweeping hypothesis [9] is valid in the inner region, where the convective flow is approximately homogenous and isotropic with a zero mean velocity.

Figure 2(b) shows the measured $C_{T}(r, \tau)$ as a function of $\tau$ for different values of $r$ in the outer region, in which the peak value of $C_{T}(r, \tau)$ decays from 0.5 to the noise level. In contrast to the situation in the inner region, the peak position $\tau_{p}$ now starts to increase with $r$ and the shape of the measured $C_{T}(r, \tau)$ becomes more asymmetric with respect to $\tau_{p}$ for large values of $r$. These changes suggest that the velocity (and hence temperature) fluctuations become inhomogeneous over large separations $r$. It has been shown [3] that in the aspect-ratio-1 cell LSC is not exactly of circular shape (which is true only in the central core region). Rather, the rotation is around a tilted ellipse with its long major axis aligned along the diagonal of the upright cylinder. Such a tilted LSC will give rise to a small horizontal mean velocity $U(r)$ when the separation $r$ becomes large (in the outer region). The value of $U(r)$ will increase when the measuring position is moved further toward the sidewall. We believe that this small horizontal velocity is the cause for the peak position $\tau_{p}$ to become nonzero and increase with $r$.

The asymmetry of the measured $C_{T}(r, \tau)$ in the outer region can be attributed to the asymmetric shape of thermal plumes. It has been shown [12] that warm (cold) plumes produce positive (negative) clifflike spikes in the temperature time series data. In the inner region, warm and cold plumes are fully mixed and the clifflike asymmetry in temperature fluctuations are averaged out in the measured $C_{T}(r, \tau)$. Thus $C_{T}(r, \tau)$ has a symmetric shape with respect to $\tau_{p}$. In the outer region, however, there are more warm (or cold) plumes accumulated in the sidewall region, making the temperature signal have more positive (or negative) clifflike fluctuations. Such an asymmetric distribution of temperature fluctuation will result in an asymmetric temporal correlation function $C_{T}(r, \tau)$ in $\tau$ (at a fixed value of $r$ ).

By taking the position dependence of temperature fluctuations into consideration, we introduce two $r$-dependent velocities, $U(r)$ and $V(r)$, in both Eqs. (3) and (4). Because the $r$ dependence is quite weak, we determine $U(r)$ and $V(r)$ iteratively. First, we fix $V(r)$ at the constant value $V_{0}=8.5$ $\mathrm{mm} / \mathrm{s}$ obtained in the inner region and find $U(r)$ from the measured $\tau_{p}$ vs $r$ curve (not shown) using Eq. (4). The resulting $U(r)$ is shown in Fig. 3. It is found that the obtained $U(r)$ (circles) increases with $r$ and can be fit to a linear function of $r$ :

$$
U(r)=\alpha\left(r-r_{0}\right),
$$

with $\alpha=0.1 \mathrm{~s}^{-1}$ and $r_{0}=10 \mathrm{~mm}$ (lower solid line). The obtained value of $\alpha$ is close to the estimated rotational frequency of LSC, $\gamma_{u} \simeq 0.17 \mathrm{~s}^{-1}$, at $\mathrm{Ra}=2 \times 10^{10}$ [3]. With the obtained $U(r)$, we then fine tune the value of $V(r)$ so that all the measured $C_{T}(r, \tau)$ vs $\tau$ curves at different values of $r$ superimpose when they are plotted as a function of $r_{E}$ given in Eq. (3). In the scaling plot, the main effect of changing $U(r)$ is to shift the peak position $\tau_{p}$ whereas the effect of varying $V(r)$ is to adjust the width of the single peaked correlation function.

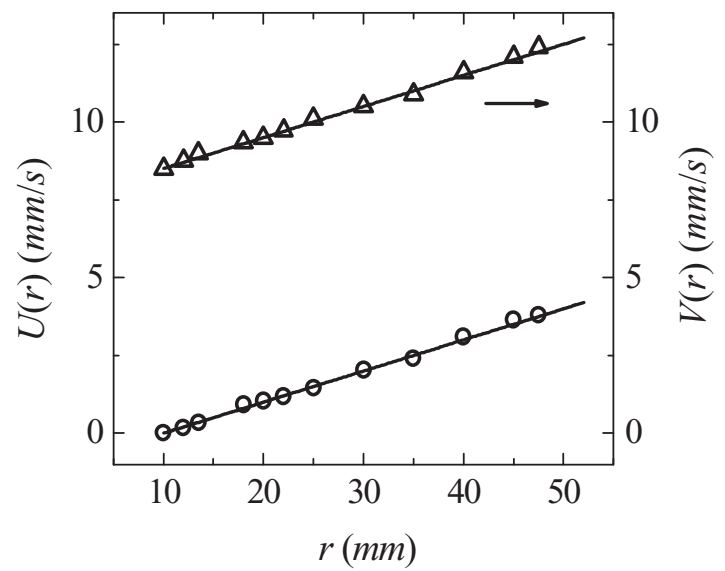

FIG. 3. Obtained $U(r)$ (circles) and $V(r)$ (triangles) as a function of $r$. The solid lines show the fitting Eq. (6) (lower solid line) and Eq. (7) (upper solid line).

The resulting $V(r)$ is also shown in Fig. 3 (triangles), which can be well fit to a linear function of $r$ as well (upper solid line):

$$
V(r)=V_{0}+\alpha\left(r-r_{0}\right) .
$$

Except for a constant offset $V_{0}$, the obtained values of $\alpha$ and $r_{0}$ for $V(r)$ are the same as those for $U(r)$.

To further test the scaling form of $C_{T}(r, \tau)$ in both the inner and outer regions, we plot, in Fig. 4, the measured $C_{T}(r, \tau)$ at different vales of $r$ as a function of the scaling variable $r_{E}$. The value of $r_{E}$ is obtained using Eq. (3) with $U=0$ and $V=8.5 \mathrm{~mm} / \mathrm{s}$ for $r$ in the inner region $(r \lesssim 10 \mathrm{~mm})$ and with Eqs. (6) and (7) for $r$ in the outer region $(r \gtrsim 10 \mathrm{~mm})$. It is seen that all the measured correlation functions collapse into a single master curve, indicating that the measured $C_{T}(r, \tau)$ 's indeed have the predicted scaling form $C_{T}\left(r_{E}, 0\right)$. For comparison, we also plot, in Fig. 4, the directly measured equal-time correlation function $C_{T}(r, 0)$ as a function of $r$ (open

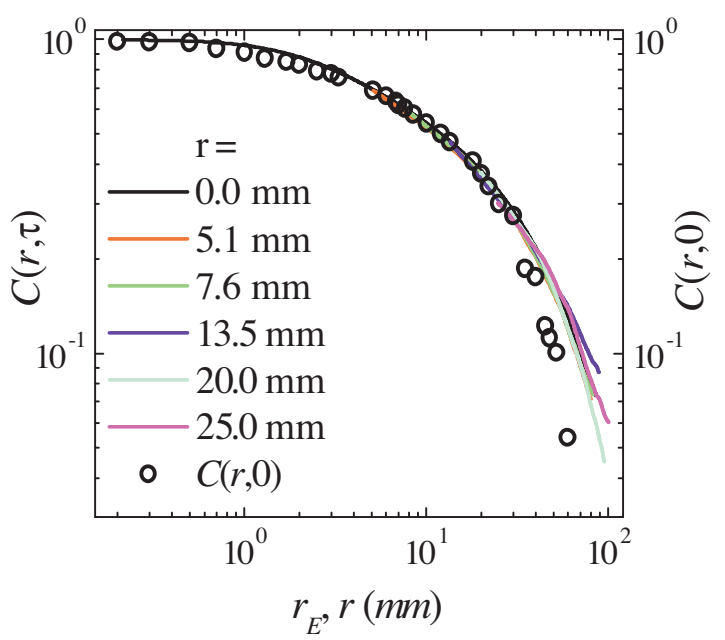

FIG. 4. (Color online) Measured $C_{T}(r, \tau)$ as a function of the scaling variable $r_{E}$ for different values of $r: 0 \mathrm{~mm}$ (black), $5.1 \mathrm{~mm}$ (red), $7.6 \mathrm{~mm}$ (green), $13.5 \mathrm{~mm}$ (blue), $20.0 \mathrm{~mm}$ (cyan), and $25.0 \mathrm{~mm}$ (magenta). For comparison, directly measured $C_{T}(r, 0)$ as a function of $r$ is also shown (circles). 


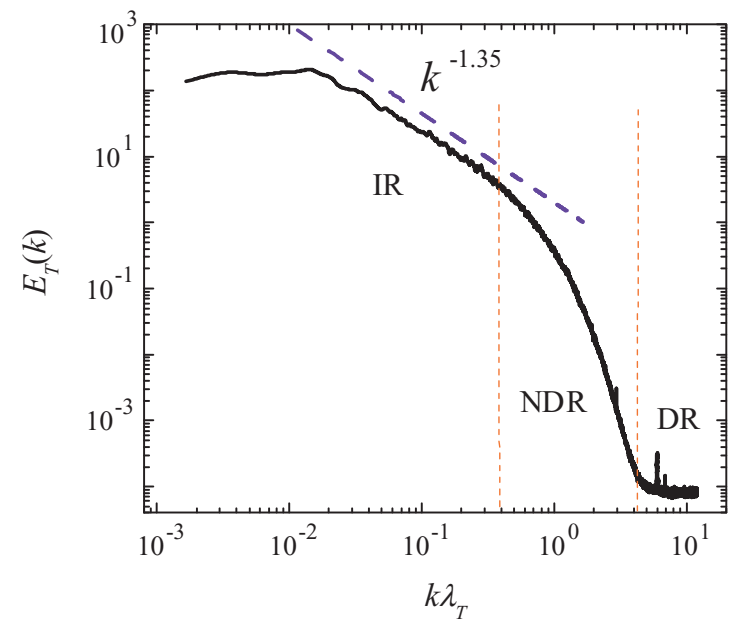

FIG. 5. (Color online) Temperature power spectrum $E_{T}(k)$ as a function of $k \lambda_{T}$. The (blue) dashed line indicates the power law, $E_{T}(k) \sim\left(k \lambda_{T}\right)^{-1.35}$. The vertical (red) dashed lines indicate the three subranges: dissipation range (DR), near-dissipation range (NDR), and inertial range (IR).

circles). In the central core region $(r \lesssim 30 \mathrm{~mm})$, the required condition of spatial homogeneity for the scaling theory to hold is satisfied and thus the measured $C_{T}(r, 0)$ and $C_{T}\left(r_{E}, 0\right)$ superpose together. For $r \gtrsim 30 \mathrm{~mm}$, however, this condition is no longer satisfied and thus the measured $C_{T}(r, 0)$ becomes systematically smaller than $C_{T}\left(r_{E}, 0\right)$. The introduction of the $r$ dependence in $U(r)$ and $V(r)$ provides a correction to the scaling form of $C_{T}\left(r_{E}, 0\right)$, but the measured $C_{T}(r, 0)$ is still affected by the spatial inhomogeneity of the flow.

An important conclusion from the above discussion is that there is no inhomogeneity correction for $r=0$, and thus one has

$$
C_{T}(0, \tau)=C_{T}\left(r_{E}, 0\right),
$$

with $r_{E}=V_{0} \tau$. With Eq. (8), one can directly obtain $C_{T}\left(r_{E}, 0\right)$, or its Fourier transform $E_{T}(k)$, from a single-point temperature time series measurement at the cell center. Figure 5 shows the temperature power spectrum $E_{T}(k)$ as a function of $k \lambda_{T}$, which is obtained using the high-resolution temporal data. In the plot, we used the Taylor microscale $\lambda_{T}$ as the normalization length. Using the equation [10] $C_{T}\left(r_{E}, 0\right) \simeq 1-\left(r_{E} / \lambda_{T}\right)^{2}$ for small values of $r_{E}$, we obtain $\lambda_{T} \simeq 3.7 \mathrm{~mm}$ directly from the compensated plot of $C_{T}\left(r_{E}, 0\right)+\left(r_{E} / \lambda_{T}\right)^{2}$ vs $r_{E}$ (not shown). The corresponding Reynolds number is $\operatorname{Re}_{\lambda_{T}} \simeq V_{0} \lambda_{T} / v \simeq$ 200.

Similar to the situation near the sidewall [1], the measured $E_{T}(k)$ reveals three distinct subranges of length scales: the inertial range, IR $\left(k \lambda_{T} \lesssim 0.4\right)$, the near dissipation range, NDR $\left(0.4 \lesssim k \lambda_{T} \lesssim 4\right)$, and the dissipation range, DR $\left(k \lambda_{T} \gtrsim 4\right)$. These three ranges are marked by the vertical (red) dashed lines in Fig. 5. While the single-point temperature power spectrum $E_{T}(f)$ has been measured previously at the cell center $[13,14]$, its connection to $E_{T}(k)$ has never been firmly established in turbulent convection [15]. With the support of the scaling results shown in Figs. 4 and 5 provides the first direct confirmation of $E_{T}(k)$ with the horizontal axis correctly labeled in units of $\lambda_{T}$. At $\mathrm{Ra}=2 \times 10^{10}$, we obtain the longest IR range, in which $E_{T}(k)$ shows a power-law scaling:

$$
E_{T}(k) \sim\left(k \lambda_{T}\right)^{-\gamma}
$$

with $\gamma=1.35 \pm 0.05$ [(blue) dashed line]. A similar scaling result was obtained previously in the convection systems of low-temperature helium gas [13] and water [16,17]. The obtained value of $\gamma$ is slightly smaller than the Corrsin-Oboukhov value of $\gamma=5 / 3$ for passive scalars in a turbulent flow at sufficiently large Reynolds numbers [6]. Possible reasons for this deviation have been discussed elsewhere $[18,19]$.

\section{ACKNOWLEDGMENTS}

We have benefited from illuminating discussions with G.-W. He. This work was supported by the Hong Kong RGC under Grant No. HKUST-603208.
[1] X.-Z. He, G. He, and P. Tong, Phys. Rev. E 81, 065303(R) (2010).

[2] The Rayleigh number is defined as $\mathrm{Ra}=\alpha g \Delta T H^{3} /(\nu \kappa)$, where $g$ is the gravitational acceleration, $\Delta T$ is the temperature difference across the fluid layer of thickness $H$, and $\alpha, v$, and $\kappa$ are, respectively, the thermal-expansion coefficient, the kinematic viscosity, and the thermal diffusivity of the fluid. The Prandtl number is defined as $\operatorname{Pr}=v / \kappa$.

[3] X.-L. Qiu and P. Tong, Phys. Rev. E 64, 036304 (2001).

[4] E. Calzavarini, F. Toschi, and R. Tripiccione, Phys. Rev. E 66, 016304 (2002)

[5] C. Sun, Q. Zhou, and K. Q. Xia, Phys. Rev. Lett. 97, 144504 (2006).

[6] H. Tennekes and J. L. Lumley, A First Course in Turbulence (MIT Press, Cambridge, MA, 1999).

[7] G. I. Taylor, Proc. R. Soc. London, Ser. A 164, 476 (1938).
[8] T. Burghelea et al., Phys. Fluids 17, 103101 (2005).

[9] R. H. Kraichnan, Phys. Fluids 7, 1723 (1964).

[10] G.-W. He and J.-B. Zhang, Phys. Rev. E 73, 055303(R) (2006); X. Zhao and G.-W. He, ibid. 79, 046316 (2009).

[11] X.-Z. He and P. Tong, Phys. Rev. E 79, 026306 (2009).

[12] S.-Q. Zhou and K. Q. Xia, Phys. Rev. Lett. 89, 184502 (2002).

[13] B. Castaing et al., J. Fluid Mech. 204, 1 (1989).

[14] F. Chillá et al., Europhys. Lett. 22, 23 (1993).

[15] D. Lohse and K.-Q. Xia, Annu. Rev. Fluid Mech. 42, 335 (2010).

[16] Y.-B. Du and P. Tong, Phys. Rev. E 63, 046303 (2001).

[17] S.-Q. Zhou and K.-Q. Xia, Phys. Rev. Lett. 87, 064501 (2001).

[18] X. Chavanne et al., Phys. Fluids 13, 1300 (2001).

[19] Y. X. Huang et al., Phys. Rev. E 82, 026319 (2010). 